\title{
Prevalence of anemia and iron deficiency in patients with pancreatic ductal adenocarcinoma (PDAC) initiating systemic therapy
}

L. Kraj ${ }^{1}$ MD, PhD, M. Osmola MD1ㄴ. K. Woźniak MD11, G. W. Basak MD, PhD¹, J. Sierdziński PhD²,

A. Hoffman MD'1, M. Maciejak ${ }^{1}$, A. Waszczuk-Gajda MD, PhD ${ }^{1}$

CONTACT: mal.osmola@gmail.com

${ }^{1}$ Department of Hematology, Oncology and Internal Diseases, Medical University of Warsaw, Poland.

2 Department of Medical Informatics and Telemedicine, Medical University of Warsaw, Poland.

\section{INTRODUCTION:}

Anemia and iron deficiency (ID) are common findings and complications of therapy in cancer patients.

Limited data are available regarding the incidence and causes of anemia in patients with PDAC.

The aim of this study was to evaluate the incidence of anemia and the deficiency of micro-nutrients necessary for proper hematopoiesis

\section{MATERIALS AND METHODS:}

- A retrospective, single-center analysis of 82 patients diagnosed with PDAC were consecutively subjected to initiate systemic chemotherapy.

- Complete blood count, iron status, vitamin B12 and folate concentrations were assessed prior to the onset of systemic treatment.

- Study definitions can be found below (Table 1.)

\section{RESULTS:}

$\checkmark 62$ patients $(75.6 \%)$ were qualified for palliative and $20(24.4 \%)$ for adjuvant chemotherapy

Anemia (hemoglobin $<12 \mathrm{~g} / \mathrm{dl}$ ) was noted in $40.1 \%$ of patients, $(43.5 \%$ in palliative and $30 \%$ in the adjuvant group)

$\checkmark$ Iron deficiency was noted in $46 \%$ of patients $(51,5 \%$ in palliative and $30 \%$ in the adjuvant group)

$\checkmark$ FID occurred twice more often than AID constantly in every group (Picture 1.)

Vitamin B12 and folate deficiency were rare findings (Table 1.)

$\checkmark$ Interestingly, 33\% of patients from the palliative group had elevated levels of vitamin B12, whereas in the adjuvant group it was not observed (Picture 2.)

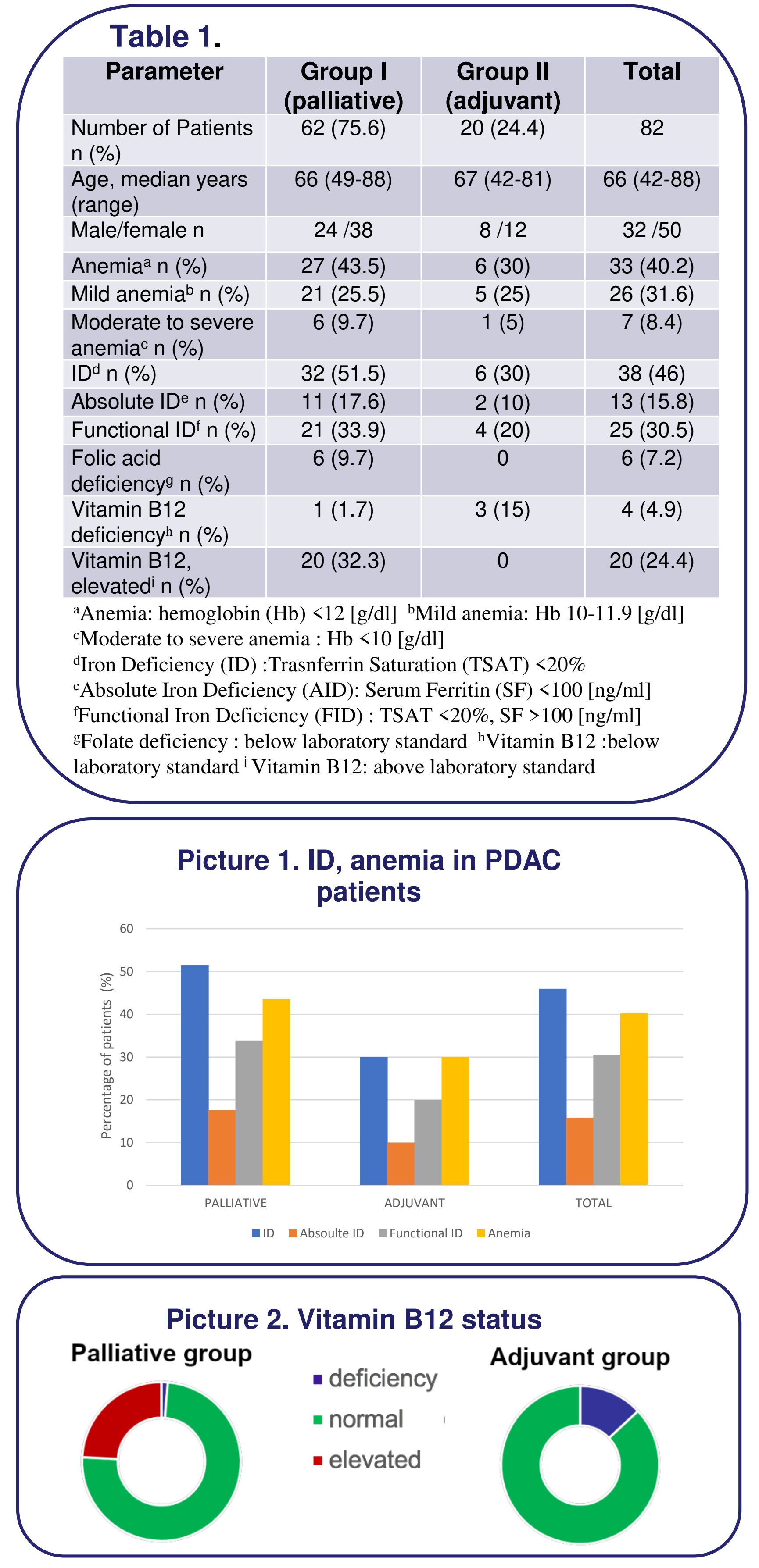

CONCLUSIONS:

Anemia and ID are frequently noted in patients with PDAC before systemic therapy (Pic. 1.) B12/folate deficiency is a rare cause of anemia in PDAC (Pic. 2.)

Iron status should be carefully assessed in the management of PDAC patients qualified for the chemotherapy.

The importance of serum vitamin B12 elevation among patients with PDAC requires further research. 